Meat Science Volume 167September 2020 Article number 108161

\title{
Multifunctional betanin nanoliposomes-incorporated gelatin/chitosan nanofiber/ZnO nanoparticles nanocomposite film for fresh beef preservation
}

Sajed Amjadi ${ }^{\mathrm{a}}$, Maryam Nazari ${ }^{\mathrm{b}}$, Safar Ali Alizadeh $^{\mathrm{c}}$, Hamed Hamishehkar $^{\mathrm{d}}$

${ }^{a}$ Department of Food Science and Technology, Faculty of Agriculture, Urmia University, P.O. Box 57561-51818, Urmia, Iran

${ }^{\mathrm{b}}$ Biotechnology Research Center, Tabriz University of Medical Sciences, Tabriz, Iran

${ }^{c}$ Department of microbiology, Qazvin University of Medical Sciences, Qazvin, Iran

${ }^{\mathrm{d}}$ Drug Applied Research Center, Tabriz University of Medical Sciences, Tabriz, Iran

\begin{abstract}
The objective of this study was to fabricate betanin nanoliposomes incorporated gelatin/chitosan nanofiber/ZnO nanoparticles bionanocomposite film (G/CH NF/ZnO NPs/B NLPs) and investigate its effects on the preservation of fresh beef. The scanning electron microscopy image of nanocomposite film displayed a good inter-connective porous morphology. Fourier transform infrared and X-ray diffraction analysis confirmed the formation of new hydrogen bonds and enhanced crystallinity through the addition of CH NF, ZnO NPs, and B NLPs. The G/CH NF/ZnO NPs/B NLPs film exhibited satisfactory mechanical properties and high surface hydrophobicity (water contact angle $=92.49 \pm 3.71^{\circ}$ ). The incorporation of ZnO NPs and B NLPs in the nanocomposite film provided high antibacterial activity and DPPH inhibition activity (53.02 \pm $3.26 \%$ ). The growth of inoculated bacteria, lipid oxidation, and the changes in the $\mathrm{pH}$ and color quality of the beef samples were controlled by packaging with the fabricated film. In conclusion, the G/CH NF/ZnO NPs/B NLPs nanocomposite has a high potential for meat preservation
\end{abstract}

